\title{
Development process and testing of partial discharge detection device on medium voltage XLPE cable
}

\author{
Mohamad Izmir Farhan Mohamad $\operatorname{Radzi}^{1}$, N H Nik Ali ${ }^{2}$, Azrul Mohd Ariffin ${ }^{3}$, \\ Muhamad Safwan Abd Rahman ${ }^{4}$, Norhidayu Rameli ${ }^{5}$, Mohamad Radzi Ahmad ${ }^{6}$, \\ Ali Syari'ati Mohd Salleh ${ }^{7}$ \\ ${ }^{1,2,3,4}$ Institute of Power Engineering, Universiti Tenaga Nasional, Malaysia \\ ${ }^{5}$ TNB Research, Kawasan Institusi Penyelidikan, Malaysia \\ ${ }^{6}$ Department of Electrical Electronics Engineering, Universiti Teknologi Petronas, Malaysia \\ ${ }^{7}$ Faculty of Computer Science, Universiti Teknologi Malaysia, Malaysia
}

\begin{tabular}{l}
\hline Article Info \\
\hline Article history: \\
Received Jan 20, 2020 \\
Revised Mar 23, 2020 \\
Accepted Apr 7, 2020 \\
\hline
\end{tabular}

\section{Keywords:}

Acoustic emission

Cross-linked polyethylene

Medium voltage

Partial discharge

Ultrasonic sensor

\begin{abstract}
High voltage assets play a vital role in providing uninterrupted power to the consumers and any slight problems experienced by the assets may cause losses in millions of dollars to businesses. Therefore it is of utmost importance to monitor the health of high voltage assets. This research presents the development process of a Partial Discharge (PD) device that is able to detect PD acoustic waves for monitoring high voltage assets purposes. Medium voltage Cross-Linked Polyethylene (XLPE) cable was used which was introduced with spherical void defects at the joints of the cable that functioned to produce PD acoustic waves. Outcome of the development processes provides the finished design of the PD sensing device, known as Partial Discharge Detection (PDD) device. The functionality of the PDD device was also assessed through controlled experimentations, and they proved to be successful. Pure PD waveform captured by the ultrasonic sensor was similar when compared to a HFCT sensor's pure PD waveform. The PDD device is a small and affordable, and is opened to various improvements such as integrating Artificial Intelligence (AI) unto the device, and one day may replace most existing bulky and expensive PD sensing devices that are readily available in the market.
\end{abstract}

Copyright $@ 2020$ Institute of Advanced Engineering and Science. All rights reserved.

\section{Corresponding Author:}

Mohamad Izmir Farhan Mohamad Radzi, Institute of Power Engineering,

Universiti Tenaga Nasional, Jalan IKRAM-UNITEN, 43000, Kajang, Selangor, Malaysia.

Email: Mohamad.izmir.farhan@gmail.com

\section{INTRODUCTION}

Power cables are considered to be an important asset. Power cables are not just being utilized in electrical power transmission but are also used under various environmental and mechanical conditions [1]. Under all these utilization fields of power cables, 'Degradation' occurs in power cables. Normally, PD is the main culprit for the degradation in power cables. PD is a natural breakdown phenomenon of a minute area of insulation which is subjected to high voltage [2]. In a detailed perspective, PD occurs adjacent to a conductor when the insulation between conductors do not completely bridge each other [3]. Aging assets, faulty design, and material defects are usually the main causes which can eventually lead to the production of various sources of PD $[4,5]$. In this paper, internal partial discharge in the form of void discharge will be of the main focus in which the air voids are located near to the insulation screen of the XLPE cable. Void discharge is a common type of internal PD which commonly occurs in medium voltage XLPE cables that have joint defects [6, 7]. 
The development of high rated voltage for XLPE cable systems is commonly used in meeting the increasing demand of high power transmission fields $[8,9]$. Since delivering efficient power to consumers revolves heavily around the XLPE cables' health, continuous observation of their health is crucial. PD damages the health of XLPE cables through the degradation of insulation materials, thus a PD monitoring device should be developed. PD monitoring devices are already available in the market, however, these devices are expensive and bulky $[10,11]$. Most electrical equipment produce a broad range of sound, this includes ultrasound waves which can be easily detected through basic ultrasonic testing procedures. Various methods and sensors have been developed in order to assess the ultrasonic testing [12-16]. This paper will be using ultrasonic measurement as a means of detecting internal PD due to void discharge, it is considered to be one of the best acoustic emission testing method. A High Frequency Current Transformer (HFCT) will also be used in this research for comparative purposes. The completed PDD device prototype should be able to detect internal PD in medium voltage XLPE cable. Future major accomplishment of the PDD device is that the device would be able to pose as a better option when compared to PD sensors that are already mass produced such as HFCT sensors and Acoustic Emission (AE) sensors [17-19]. Therefore, this paper's main focuses are the elaboration of the design details of a working PDD prototype and the functionality tests of the device based on controlled PD experimentations which have been studied and presented.

The rest of the paper is organized as follows: Section 2 presents the methodology of the paper, which is divided into smaller sections, whereby Section 2.1 explains the Mechanism Flowchart of PDD device, while Section 2.2, Section 2.3, and Section 2.4, explains the List of elements needed, Designing the PDD device using LT Spice and Designing the PDD device using Autodesk EAGLE respectively. Section 3 explains the Design of Experiment while Section 4 shows the extraction of pure PD waveform using MATLAB. Finally, Section 5 displays the Results and Discussions of this research paper and Section 6 presents the conclusive findings for this research paper.

\section{METHODOLOGY}

In designing the PDD device, certain necessary steps have to be taken. The steps to be followed are predesign process, defining the mechanism flowchart of PDD device, listing the required elements in designing the PDD device, designing the PDD device using LT Spice and designing the PDD device using Autodesk EAGLE.

\subsection{Mechanism Flowchart of PDD device}

A mechanism flowchart was prepared in order to fully explain the process flow of the functionality of the PDD device, and only from there can the list of electronic elements be provided.The PDD device will be positioned near a designated high voltage asset which consists of PD sources. The ultrasonic sensor on the device then detects the acoustic PD waves and captures the PD waveforms manually through the use of a digital oscilloscope. The captured PD waveforms will then be processed using MATLAB for further analyzation in order to obtain pure PD waveforms. The mechanism is based on an infinite loop process in order for the device to continuously collect raw PD waveforms and will serve as a base guideline for the whole functionality process of the PDD device. Figure 1 shows the mechanism flowchart of the PDD device.

\subsection{List of required electronic elements needed in designing the PDD device}

In designing the PDD device, the fundamental characteristics of the major electronic elements or components that are to be used must be taken into primary consideration. This is to ensure a smooth assembly process of the device is achieved in the form of a Printed Circuit Board (PCB). The list of components that were used in this research were ceramic capacitors 101 (C3 and $\mathrm{C} 2$ with values of $100 \mathrm{pF}$ each), 2mm 5kV DC Barrel Jack, resistors (R8, R7, R5 and R4 with value of $10 \mathrm{k} \Omega$, R2 with value of $1 \mathrm{k} \Omega$ and R1 and R6 with value of 330 $\Omega$ ), 3.5mm Stereo Jack with two switches (KYCON STX3100), ultrasonic sensor (C1) with frequency of $40 \mathrm{kHz}$ and sensitivity of $-63 \mathrm{~dB}$ typ. $(0 \mathrm{~dB}=10 \mathrm{~V} / \mathrm{Pa})$, trimmer potentiometer $(\mathrm{R} 3)$ with value of $5 \mathrm{k} \Omega$ and integrated circuit $\mu \mathrm{A} 741 \mathrm{CP}$ (IC4 and IC3). These components will be orderly assembled into a PCB form, where the design parameters and specifications of the PDD device can easily be entered using Autodesk EAGLE software.

\subsection{Designing the PDD device using LT Spice}

The PDD device comprises of an ultrasonic sensor, which enables itself to detect acoustic sound waves. This device is designed only for the sole purpose of having to detect a real PD waveform produced by the void discharge in the medium voltage XLPE cable. For the first process, a receiver circuit is created, which serves the purpose of consisting of an ultrasonic sensor. In this step it is important to detect the ultrasonic signal emitted by the PD source, so that the captured signal will go through a differential amplifier. The function of the differential amplifier is to roughly differentiate and amplify the captured signal with respect to a varied gain. The PDD device mainly revolves around the utilization of a high pass filter and a differential amplifier. In laymen's term, an ideal differential amplifier will normally provide an output voltage with respect to ground, 
which is commonly some gain times the two input voltage's differences. The above statement is explained based on the following basic formula which importantly serves as a main basis for the first pre-design process:

$$
V_{\text {out }}=A\left(V_{a}-V_{b}\right)
$$

As shown in (1) shows that $A$ is defined as the differential gain and both $V_{a}$ and $V_{b}$ are voltages with respect to ground. A software known as LT Spice was wholly used throughout the first pre-design process in order to construct the circuit design of the differential amplifier. The Differential Amplifier helps to differentiate which signal is of ultrasonic range and which is not due to the void discharge from the PD source. The differential amplifier circuit consists of two $330 \Omega$ resistors, two $10 \mathrm{k} \Omega$ resistors, one $100 \mathrm{pF}$ capacitor, a $5 \mathrm{k} \Omega$ variable resistor with adjustable gain, one 741 operational amplifier and a buzzer. The basic formula for a functional differential in obtaining the value of $V_{\text {out }}$ is as shown below:

$$
V_{\text {out }}=\left(\frac{R_{2}}{R_{1}}\right)\left(V_{2}-V_{1}\right)
$$

As shown in (2) shows that $A$ from in (1) is expressed as $\left(R_{2} / R_{l}\right)$ while $V_{a}$ and $V_{b}$ from in (1) are expressed as $V_{2}$ and $V_{l}$ as shown in (2). For the second functional process, a high pass filter circuit must be created. By definition, a high pass filter is a filter that allows only high frequency signals to be able to pass through. With this, unwanted signals such as lower frequency signals can be easily removed, which ease the classification process of the captured raw PD signal. The High Pass Filter only allows high frequency signals to pass through, inevitably removing unwanted lower frequency signals. The High Pass Filter circuit diagram consists of two $10 \mathrm{k} \Omega$ resistors, a $1 \mathrm{k} \Omega$ resistor, a $100 \mathrm{pF}$ capacitor, and a 741 operational amplifier. The $V_{\text {out }}$ is the filtered raw PD signal, which is later to be processed and analyzed using MATLAB. These two finished circuit designs has to be combined in order to finally form the completed PDD device circuit diagram. The circuit designs can be viewed in a previous research paper [10]. With this completed final circuit design of the PDD device, this exact design can be exported into Autodesk EAGLE software. This is to ensure that the same circuit design can be converted into a Printed Circuit Board (PCB) format. Only with this PCB format can the device be fabricated into a physical form.

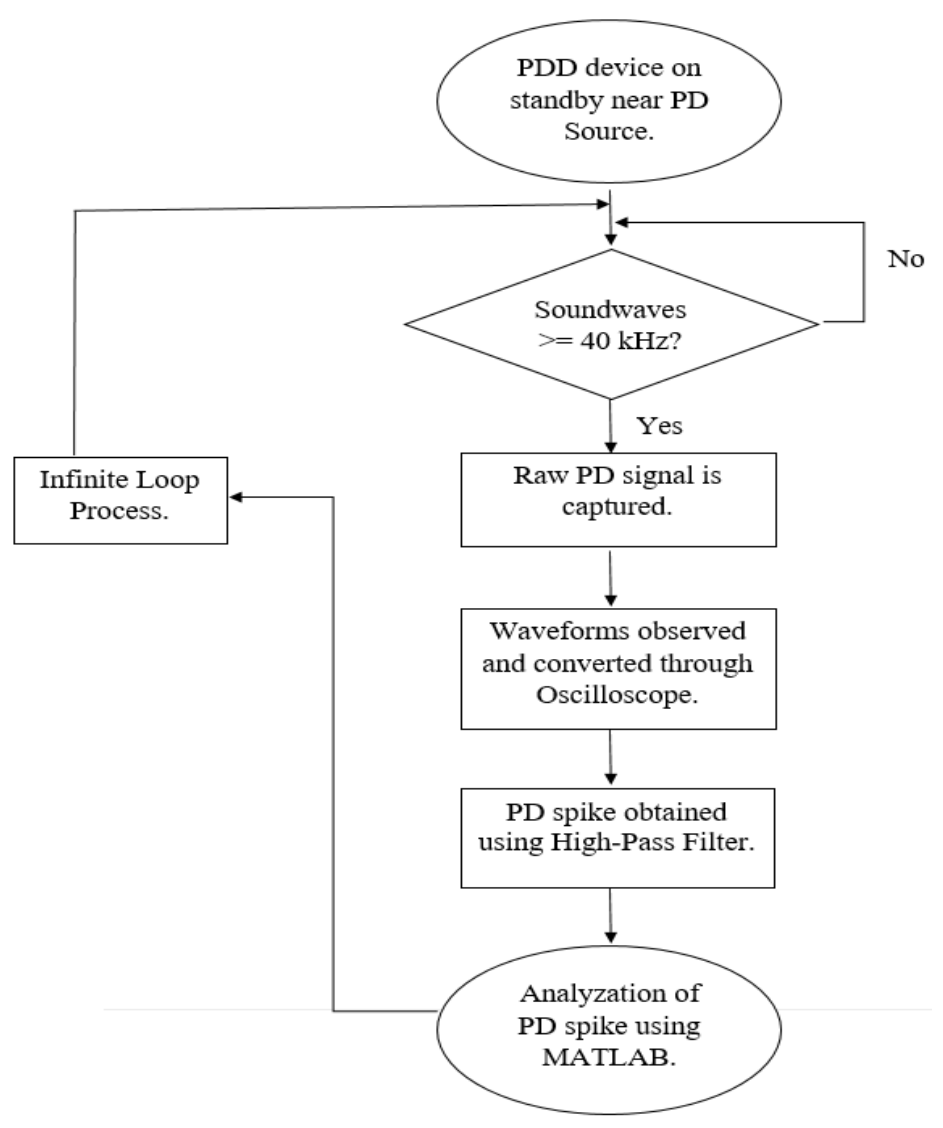

Figure 1. Design flowchart of the PDD device 


\subsection{Designing the PDD device using Autodesk EAGLE}

Exporting the final circuit diagram from LT Spice into Autodesk Eagle enables the diagram to be converted into a schematic diagram. The electronic components were carefully selected and inserted into the workspace provided in Autodesk EAGLE. Arrangement of the chosen electronic components were done in an orderly manner and the 'Net' action tab was used to connect all the electronic components. With that done, the software will automatically connect all the components together in order to establish secure connections between the components. Next, the conversion of the established schematic diagram into a PCB diagram allows the PCB to be designed in the shortest possible route specifically for the 'Via'. A 'Via' functions to establish connections between the bottom and top layer of the PCB. The PDD device uses a simple single layer circuit board, where only one copper layer is visible on the bottom side of the PCB. Air wires that are visible in the PCB diagram are actually guides for the connections of the PDD device's electronic components.

When converting from schematic diagram into PCB diagram some minute arrangements must be done unto the electronic components by dragging them in the desired design specifications. Next, the ground area of the PCB diagram has to be defined. This is actually done to ground the area that has no air wires or connections in order to avoid any unwanted interferences during the experimentation of the PDD device, which ultimately helps in improving the quality of the PD waveforms that are to be captured. For the net class details of the PCB diagram, the suitable values that are to be keyed in the software are $12 \mathrm{~mm}$ for the width, $20 \mathrm{~mm}$ for the drill and $10 \mathrm{~mm}$ for the clearance. To minimize the complexity of the design, the net class values were keyed in using the values provided which enable the design of a single layered PCB with a copper layered bottom for secure electronic connections. By carefully following the procedures in creating the PCB diagram, the end result is that in the form of secured connections of the air wires that are connected together simultaneously with respect to the shortest and simplest available route. The air wire connections are better suited in a more refined light blue color. This allows better indication of the PCB having established successful connections. This finished design will be fabricated by PCB manufacturers and can be viewed in a previous research paper [10].

\section{DESIGN OF EXPERIMENT (DOE)}

It is time to test the functionality of the device, through controlled experimentations. As stated above in the methodology section, the source of the PD is due to void discharge. To create the void discharge, holes are being introduced at the joints of the medium voltage XLPE cable. These holes serves as air pockets which then allows the cable joints to be considered as 'damaged' or contaminated, in order to allow joint failure. The joint failure will promote PD to occur, which therefore then allows the PDD device to be able to detect and captured the acoustic waves produced by the PD. The specifications of the holes created for the void discharge, are that the depth is 1.15 millimeters, the width is 2.93 millimeters, the shape construct is in spherical form, the permittivity of air which has a value of 1 and lastly, the void discharge is located on the right and left side of the joints of medium voltage XLPE cable which is very near to the insulation screen. With these specifications, a simulation of electric field strength in order to determine the value of PD emitted due to the void discharge can be done using a software known as Finite Element Method Magnetics (FEMM). This FEMM software aids in constructing a cutout version of the medium voltage XLPE cable used in this research.

Since it is understood that the electric field strength is directly proportional to the magnitude of PD created, it is safe to say based on the simulation done, the void discharge is sufficient enough to produce an abundant magnitude of PD. In theory, the electric field strength and an insulation's current density are known to be the highest when it is near the conductor and becomes significantly weaker when it is further away from the conductor [20, 21]. For the actual experimentation, the PDD device is placed horizontally near to the PD source, which is at the joints of the medium voltage XLPE cable. The device is supplied with a 5V DC voltage supply continuously in order to function and detect acoustic soundwaves. An AC voltage supply is used to inject subsequent amount of voltages into the medium voltage XLPE cable. The AC voltage varies from $2.5 \mathrm{kV}$ until $15 \mathrm{kV}$. Any higher than $15 \mathrm{kV}$ will propose danger to the safety of the lab's environment. When injecting AC voltage into the cable, the defect in the cable joints causes void discharges to occur and therefore, releasing acoustic waves that can be translated into PD waves. These PD waves is detected by the ultrasonic sensor on the PDD device, and converted into electrical signals. The electrical signals flow to an oscilloscope, and this oscilloscope functions in directly capturing raw PD waveforms. The oscilloscope is that of a Teledyne Lecroy oscilloscope with a bandwidth of $500 \mathrm{MHz}$, a sampling rate of $2.5 \mathrm{GS}$ respectively and uses a $50 \mathrm{~Hz}$ filter. In this experimentation, a commercial PD sensor known as a HFCT is also used to detect the acoustic soundwaves of the PD source. This is purely for comparison purposes in order to justify the functionality of the PDD device. The PD waveforms from the two sensors captured by the oscilloscope will be further analyzed using MATLAB. 


\section{PURE PD WAVEFORM EXTRACTION USING MATLAB}

Raw PD waveforms contains unwanted signals such as noise, and the extraction process helps to remove the interferences in order to realize the comparison of both sensor's data. The first step process is to convert the raw PD waveforms captured using the digital oscilloscope into excel file type format. These excel version of the waveforms will then be converted again into a new file type format known as MATLAB files format (.mat). This is basically known as the initial pre-processing stage of the extraction process. Next, DC offset from the raw data is removed for further processing. The reason why DC offset is removed from the raw data is because the raw signals that are captured using both sensors already consist of the production of DC offset. The main culprit for the production is due to the operation of the pulse generation hardware during the experimentation. In order to remove the DC offset, the mean amplitude of the entire cycle was subtracted from one of each individual sample. By doing this, each of the individual sample will be able to exhibit a zero mean [22, 23]. Although the experimentations were done in a low noise level controlled laboratory condition, background noises still have to be removed before exerting the extraction process unto the raw PD waveforms. This process is known as de-noising which can be used to comply the task of noise suppression, and such techniques are known as Fast Fourier Transform (FFT) based de-noising and low pass filtering. A method known as hard-thresholding was carried out to pre-extract the PD pulses after de-noising process. The value that was set for hard-thresholding is set to $1.5 \mathrm{mV}$. For the actual extraction process, a famous algorithm called peak detection technique is used. The technique was executed unto the raw data, transferring each pulse into a new variable matrix. This new variable matrix replaces the removed pulses with a zero vector $[22,23]$.

The peak detection technique process was done several more times in order to ensure that the peak magnitude of the remaining pulses in the raw data is much smaller than the pre-defined threshold value. Then only can the PD pulses be isolated from unwanted background noises. The threshold value however cannot be set too high, as a large possibility of information loss in PD pulses may likely to occur during the process. On the contrary, if the threshold value is set too low, PD pulses might be mistakenly interpreted from unwanted noise pulses [24-26]. The final step process is the Pulse Pairing Process. This process is carried out when the peak magnitude of a PD pulse is much smaller than the stated pre-defined threshold value. This process is also done based on a reasonable time of flight duration of the PD pulses. In the end, these pure PD pulses that are obtained from both PDD device and HFCT are then measured and compared based on their time domain.

\section{RESULTS AND DISCUSSIONS}

The results that were obtained after going through all the stated process in obtaining pure PD pulses are as expected. The pulse pairing process of the PD pulses from both sensors can be seen in Figure 2. Note that the voltage chosen for the display of results are picked from the best amongst the pairs that are obtained. It can be simply seen that the PD pulses detected by the PDD device are similar to that of the PD pulses detected by the HFCT sensor. This proves that the PDD device is working perfectly as expected and that the functionality of the device is justified by comparing it to the HFCT. There is without a doubt that the PDD device functions just as how the HFCT would in terms of detecting PD pulses. Figure 2 displays the orange color to be the PDD device's PD pulse while the blue color is stated to be the HFCT's PD pulse.

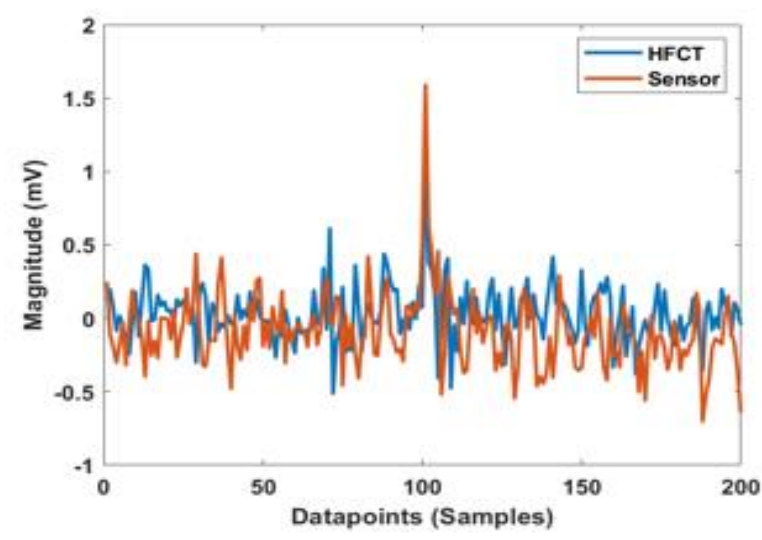

(a) $6.5 \mathrm{kV}$

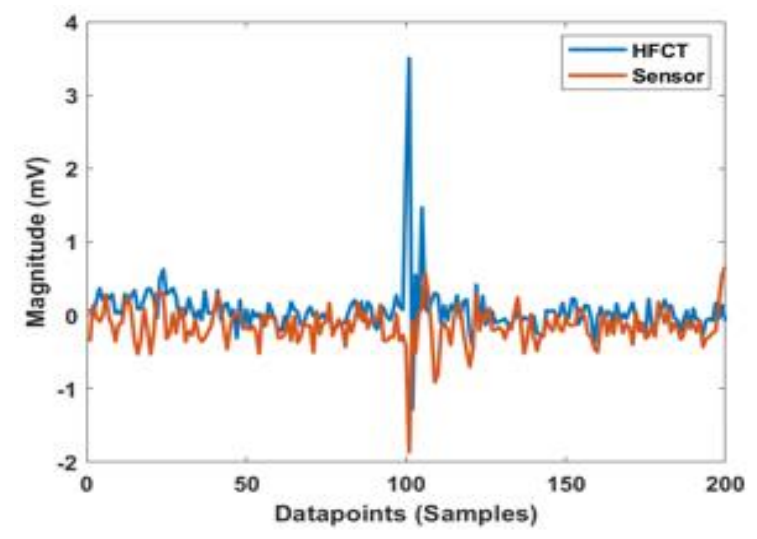

(b) $7.5 \mathrm{kV}$ 


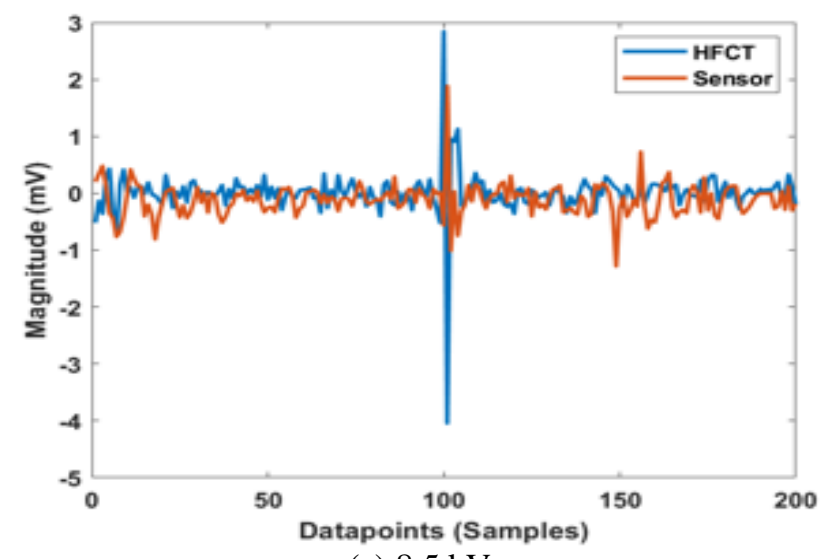

(c) $8.5 \mathrm{kV}$

Figure 2. Pulse pairing process of PD pulses captured using both PDD device and HFCT sensor

From Figure 2, it can be justified that the PDD device does detect pure PD pulses much like the HFCT sensor did. However, it can also be observed that the magnitude of the PD pulses detected by the PDD device is quite smaller as compared to the HFCT sensor. This is naturally due to the nature of acoustic emission sensing method by using an ultrasonic sensor itself, whereby the method is abruptly affected by extraneous noise and signal attenuation tends to happen frequently. HFCT sensor is not affected by these factors so to say, hence the difference in the magnitude of electric field strength.

As for the FEMM simulation of the spherical void defect, the magnitude of the electric field strength from the simulation is found out to be approximately $3 \mathrm{MV} / \mathrm{m}$. This value is indeed a high value for production of PD. Since electric field strength is directly proportional to the magnitude of PD, it can be stated that the artificial void discharge caused by the spherical void defect is more than enough to provide abundant PD which then leads to high production of acoustic soundwaves. This in return helps ease both the sensors to detect easily the soundwaves and capture abundant PD waveforms using the digital oscilloscope. The electric field strength decreases as it is further away from the spherical void defect. The magnitude values of PD pulses captured by the PDD device are very much dependent on the magnitude of AC voltage applied during the experimentation. The higher the magnitude of the injected AC voltage, the higher the magnitude of the PD pulses. Table 1 shows the maximum average magnitude of the PD pulses obtained by the PDD device for each different voltage applied.

Table 1. PD pulses maximum average magnitude captured by PDD device

\begin{tabular}{cc}
\hline $\begin{array}{c}\text { Voltage } \\
\text { Applied }(\mathrm{kV})\end{array}$ & $\begin{array}{c}\text { Maximum Average Magnitude } \\
(\mathrm{mV})\end{array}$ \\
\hline 6.5 & 0.1017 \\
7.5 & 0.4024 \\
8.5 & 0.6981 \\
\hline
\end{tabular}

Although the PDD device falls behind the HFCT sensor in terms of the accuracy of capturing PD pulses, ultimately, the device is still able to achieve the main objective of this research and that is to detect PD pulses in medium voltage XLPE cable due to internal void discharges. Advantages of the PDD device compared to the HFCT sensor are such as costing a lot less as compared to buying one whole set of HFCT sensor, smaller and much easier to be used and opened to a wide variety of upgrades such as implementing Artificial Intelligence (Ai) which are quite hard to implement unto the HFCT sensor.

\section{CONCLUSION}

In this research paper, an intuitive insight on the meticulous design process of the PDD device is presented properly. The main objectives of this research which are to design a functional PDD device that can detect PD due to void discharge in medium voltage XLPE cable, and to assess the functionality of the PDD device through controlled experimentations have been realized and achieved successfully. Results obtained throughout the experimentations strongly infers that the PDD device is positively functioning as expected, and that the values of the magnitude of PD obtained are in the acceptable range. Furthermore, 
the pure PD waveform captured by the PDD device very much resembles the pure PD waveform captured by the HFCT sensor. PD monitoring is very crucial in maintaining the health of high voltage assets in reducing the repair and maintenance costs, and this PDD device helps proficiently in this particular field. All in all, this research much likely aids in opening new pathways for the PDD device to blossom into a more sophisticated and technology driven sensing tool.

\section{ACKNOWLEDGEMENTS}

The authors of this research study would like to honorably acknowledge TNB Research Sdn. Bhd., for their kind and generous support throughout the whole research period. Special thanks also goes to the individuals who have indirectly or directly contributed to this research project.

\section{REFERENCES}

[1] Tzimas, S. Rowland, L. A. Dissado, M. Fu, and U. H. Nilsson, "Effect of long-time electrical and thermal stresses upon the endurance capability of cable insulation material," IEEE Transactions on Dielectrics and Electrical Insulation, vol. 16, no. 5, pp. 1436-1443, 2009.

[2] M. Karimi, M. Majidi, H. MirSaeedi, M. M. Arefi and M. Oskuoee, "A novel application of deep belief networks in learning partial discharge patterns for classifying Corona, surface, and internal discharges," in IEEE Transactions on Industrial Electronics, vol. 67, no. 4, pp. 3277-3287, Apr 2020.

[3] High-voltage test techniques-Partial discharge measurements, IEC Standard 60270, BS EN Standard 60270, Electrotechnical-sector-committee, Br. Stand. Board, 2000.

[4] B. Fruth and L. Niemeyer, "The importance of statistical characteristics of partial discharge data," IEEE Transactions on Dielectrics and Electrical Insulation, vol. 27, no. 1, pp. 60-69, 1992.

[5] E. Gulski, "Digital analysis of partial discharges," IEEE Transactions on Dielectrics and Electrical Insulation, vol. 2, no. 5, pp. 822-837, 1995.

[6] G. Ma, C. Li, X. Chen, J. Jiang, Z. Ge, and W. Chang, "Numerical sensor design for partial discharge detection on power cable joint," IEEE Trans. Dielectr. Electr. Insul., vol. 22, no. 4, pp. 2311-2319, 2015.

[7] Z. Ahmed, G. A. Hussain, M. Lehtonen, L. Varacka, and J. Kudelcik, "Analysis of partial discharge signals in medium voltage XLPE cables," in 2016 17th International Scientific Conference on Electric Power Engineering (EPE), pp. 1-6, 2016.

[8] A. R. Mor, P. H. F. Morshuis, P. Llovera, V. Fuster, and A. Quijano, "Localization techniques of partial discharges at cable ends in off-line single-sided partial discharge cable measurements," IEEE Trans. Dielectr. Electr. Insul., vol. 23, no. 1, pp. 428-434, 2016.

[9] N. H. Nik Ali, M. Giannakou, R. D. Nimmo, P. Rapisarda, and P. L. Lewin, "Classification and localisation of multiple partial discharge sources within a high voltage transformer winding," in IEEE Electrical Insulation Conference (EIC), pp. 519-522, 2016.

[10] I. F. Radzi, "Partial discharge detection device using ultrasonic sensor on high voltage equipment," B. Eng. Thesis, Department of Electrical Engineering, Universiti Tenaga Nasional, Selangor, Malaysia, 2019.

[11] Yafei Zhou, Yang Qin and P. Chappell, "Cost-effective on-line partial discharge of measurements for cables," in IEEE Electrical Insulation Magazine, vol. 22, no. 2, pp. 31-38, 2006.

[12] M. Vakilian, T. R. Blackburn, R. E. James, and B. T. Phung, "Semiconducting layer as an attractive PD detection sensor of XLPE cables," IEEE Transactions on Dielectrics and Electrical Insulation, vol. 13, no. 4, pp. 885-891, 2006.

[13] A. Cavallini, G. C. Montanari, and F. Puletti, "A novel method to locate PD in polymeric cable systems based on amplitudefrequency (AF) map," IEEE Transactions on Dielectrics and Electrical Insulation, vol. 14, no. 3, pp. 726-734, 2007.

[14] M. Tozzi, A. Cavallini, G. C. Montanari, and G. L. G. Burbui, "PD detection in extruded power cables: An approximate propagation model," IEEE Transactions on Dielectrics and Electrical Insulation, vol. 15, no. 3, pp. 832-840, 2008.

[15] F. P. Mohamed, W. H. Siew, J. J. Soraghan, S. M. Strachan, and J. Mcwilliam, "The use of power frequency current transformers as partial discharge sensors for underground cables," IEEE Transactions on Dielectrics and Electrical Insulation, vol. 20, no. 3, pp. 814-824, 2013.

[16] A. Rodrigo, P. Llovera, V. Fuster, and A. Quijano, "High performance broadband capacitive coupler for partial discharge cable tests," IEEE Transactions on Dielectrics and Electrical Insulation, vol. 20, no. 2, pp. 479-487, 2013.

[17] W. Si, C. Fu and P. Yuan, "An integrated sensor with AE and UHF methods for partial discharges detection in transformers based on oil valve," in IEEE Sensors Letters, vol. 3, no. 10, pp. 1-3, Oct. 2019.

[18] Ching-Chau Su, Cheng-Chi Tai, Chien-Yi Chen, Ju-Chu Hsieh and Jiann-Fuh Chen, "Partial discharge detection using acoustic emission method for a waveguide functional high-voltage cast-resin dry-type transformer," 2008 International Conference on Condition Monitoring and Diagnosis, Beijing, pp. 517-520, 2008.

[19] L. Duan, J. Hu, G. Zhao, K. Chen, J. He and S. X. Wang, "Identification of partial discharge defects based on deep learning method," in IEEE Transactions on Power Delivery, vol. 34, no. 4, pp. 1557-1568, Aug. 2019.

[20] M. S. K. M. H. M. Sharif, N. A. M. Jamail, N. A. Othman, "Analysis of electric field and current density on XLPE insulator," International Journal of Electrical and Computer Engineering (IJECE)., vol. 7, no. 6, pp. 3095-3104, 2017.

[21] G. Parent, M. Rossi, S. Duchesne and P. Dular, "Determination of partial discharge inception voltage and location of partial discharges by means of paschen's theory and FEM," in IEEE Transactions on Magnetics, vol. 55, no. 6, pp. 1-4, Jun 2019. 
[22] N. H. Nik Ali, M. S. Abd Rahman, J. A. Hunter, P. Rapisarda, and P. L. Lewin, "Wavelet and Mathematical Morphology as the de-noising methods for PD analysis of high voltage transformer windings," 33rd Electrical Insulation Conference, EIC 2015 IEEE, pp. 214-217, 2015.

[23] N. H. Nik Ali, J. A. Hunter, P. Rapisarda, and P. L. Lewin, "Identification of multiple partial discharge sources in high voltage transformer windings," 2014 IEEE Conference on Electrical Insulation and Dielectric Phenomena (CEIDP), pp. 188-191, 2014.

[24] A. Z. Abdullah et al., "Wavelet based de-noising for on-site partial discharge measurement signal," Indonesian Journal of Electrical Engineering and Computer Science (IJEECS), vol. 16, no. 1, pp. 259-266, October 2019.

[25] I. F. Radzi, N. H. Nik Ali, N. Rameli, A. Mohd. Ariffin, M. S. Abd. Rahman, M. R. Ahmad, A. S. Salleh, "Partial discharge detection device using ultrasonic sensor on medium voltage XLPE cable," Indonesian Journal of Electrical Engineering and Computer Science (IJEECS), vol. 17, no. 2, pp. 886-894, Feb 2020.

[26] Rasha Abdul-nafaa Mohammed, Ali Nathem Hamoodi, Bashar M. Salih, "Partial discharge measurement in solid dielectric of H.V Cross-linked polyethylene (XLPE) submarine cable," Indonesian Journal of Electrical Engineering and Computer Science (IJEECS), vol. 17, no. 3, pp. 1578-1583, Mar 2020.

\section{BIOGRAPHIES OF AUTHORS}

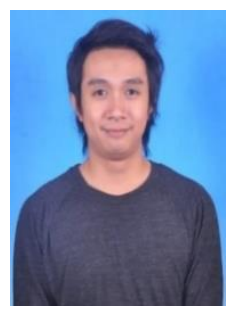

Mohamad Izmir Farhan Mohamad Radzi has successfully completed his Bachelor Degree in Electrical Power Engineering (First Class Honour), from Universiti Tenaga Nasional (UNITEN) in December 2019 fully sponsored by Yayasan Tenaga Nasional (YTN). He successfully obtained 6 semesters of Dean's List. He started his degree in Virginia Polytechnic Institute and State University in the United States of America back in 2014. He completed an industrial training program in TNB Research Sdn. Bhd and is currently working on a life assessment project for Medium Voltage Underground Cable (MVUG) under professional supervision of Assoc. Prof. Dr. Ir. Azrul bin Mohd Ariffin in conjunction with TNB Research for his Master's Degree. He previously worked as a Research Officer under the same project and supervisor. Currently, he is officially working for TNB's Distribution Network under Stakeholder Management department whilst pursuing his Master's Degree in Electrical Engineering in UNITEN.

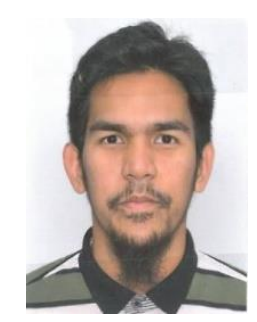

Nik Hakimi Nik Ali was born in Malaysia in 1990. He received the B.Eng. degrees in Electrical Power Engineering (First Class Honors) from the Universiti Tenaga Nasional (UNITEN), Malaysia in 2013. He received the Ph.D. degrees in Electronics and Electrical Engineering from the University of Southampton, UK in 2017. He worked as a Research Fellow in School of Electronics and Computer Science in University of Southampton, UK from 2018 to 2019. Currently, he is a Post-doctoral Researcher in Institute of Power Engineering (IPE) in UNITEN, Malaysia. He is also a graduate member of Board of Engineers Malaysia (BEM). His research interests are within the generic areas of applied signal processing. Within high voltage engineering this includes condition monitoring of high voltage cables and transformers, partial discharge measurement, HV insulation/dielectric materials, transformer rating analysis and applied signal processing.

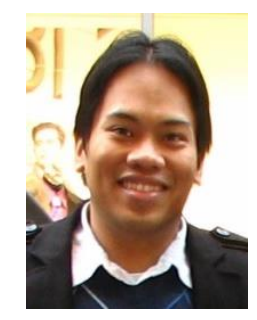

Azrul Mohd Ariffin was born in Malaysia in 1981. He received his B.Eng in Electrical Engineering (First Class Honors) from University of Southampton, UK in 2004. He then pursued his doctorate at the same university and was awarded the Ph.D degree in 2008. His thesis investigated the electroluminescence phenomenon in insulating polymers subjected high electric field. Currently, he is an Associate Professor and the Director of Programme Management Office at Universiti Tenaga Nasional (UNITEN), Malaysia. He is also a Professional Engineer (P.Eng) registered with Board of Engineers, Malaysia (BEM).

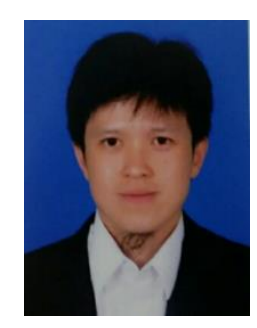

Muhamad Safwan Abd Rahman received his Master of Engineering in Electrical Power and $\mathrm{Ph} . \mathrm{D}$ in Electrical Engineering from Universiti of Southampton, United Kingdom in 2010 and 2014 respectively. He is currently a Senior Lecturer in Department of Electrical and Electronics Engineering, Universiti Tenaga Nasional (UNITEN), Malaysia. His research interests are High Voltage Engineering, Power Transformers \& Cables, Partial Discharge studies, Lightning Protection studies, Thermal Stress studies, Statistical Pattern Recognition, Finite Element Analysis using CFD \& Electrostatic Method and Insulation Coordination. He had conducted various research and consultancy works since joining UNITEN in 2014 and he is currently supervising two Ph.D candidates, 1 Master candidate and undergraduate students who are working in various fields in High Voltage. He is also actively involved in Occupational Safety and Health (OSH) activities and a member of OSH in Faculty of Engineering, UNITEN which handling safety and policies. He is also a member of IEEE and Dielectrics Electrical and Insulation Society (DEIS), a member of Institution of Engineering and Technology (IET), a member of Malaysian High Voltage Network (MyHVnet) and a member of Institute of Power Engineering (IPE), UNITEN. 

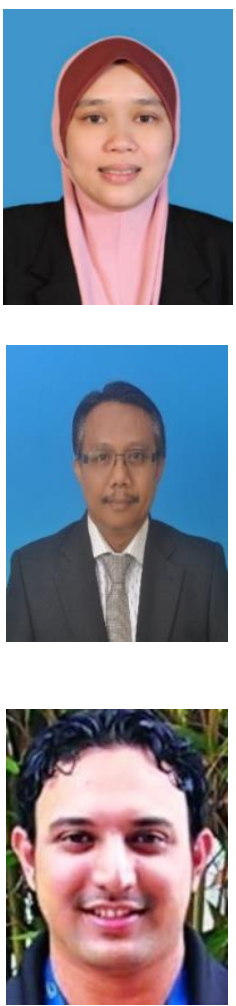

Norhidayu Rameli was graduated from Universiti Putra Malaysia with B.Eng degree in Electrical and Electronic in 2010. In 2013 and 2017, she completed her studies in MSc and PhD, respectively in Electrical Power Engineering. During MSc and PhD programmed, she had been tutoring courses including Power System labaratory, Engineering Mathematics, Electrical Circuit and involved with local and international conferences. Currently she is working in TNB Research Sdn Bhd as a researcher.

Dr. Mohamad Radzi Ahmad received his BSc. in Electrical Engineering from Purdue University School of Engineering and Technology Indianapolis USA in 1991, MSc. in Electronics System Design Engineering from Universiti Sains Malaysia in 2010, and PhD in Electrical and Electronics Engineering from Universiti Teknologi PETRONAS Malaysia in 2014. He joined Universiti Teknologi PETRONAS in 2016, where he is currently a Senior Lecturer in the Electrical and Electronic Engineering Department. Prior to joining UTP, Dr Radzi had 20 years of professional experience with electronic industries such as Intel, Avago, Western Digital and Fairchild Semiconductor, and held various positions ranging from Senior Manager to Product R\&D Engineer.

Ali Syari'ati Mohd Salleh received his BSc. in Electrical Engineering from Universiti Teknologi Mara in 2009, Msc. In Computer and Microelectronics system from Universiti Teknologi Malaysia in 2015. He joined Intel Microelectronic system as Analog SoC Validation, SoC HW Validation, IOT, Machine Learning and Deep Learning Engineer from 2009 until 2019. 